\section{Desigualdade no acesso a medicamentos para doenças crônicas em mulheres brasileiras}

\author{
Inequality in Brazilian women's access to medicines \\ for chronic diseases
}

\author{
Inequidad en el acceso a medicamentos para \\ enfermedades crónicas en mujeres brasileñas
}

\begin{abstract}
1 Programa de Pós-graduação em Organizações e Mercados, Universidade Federal de Pelotas, Pelotas, Brasil. 2 Centro Internacional de Equidade em Saúde, Universidade Federal de Pelotas, Pelotas, Brasil.

3 Programa de Pós-graduação em Epidemiologia,

Universidade Federal de

Pelotas, Pelotas, Brasil.

Correspondência F. Katrein

Rua General Teles 793, apto.

301, Pelotas, RS

96010-310, Brasil.

flaviakatrein@gmail.com
\end{abstract}

Flávia Katrein 1

Cesar Augusto Oviedo Tejada 1

Maria Clara Restrepo-Méndez 2

Andréa D. Bertoldi 3

\section{Resumo}

This study aimed to assess the prevalence of access to continuous prescription drugs for a group of chronic diseases and to investigate the existence of socioeconomic inequalities in access. The data are from the Brazilian National Demographic and Health Survey on Women and Children (2006), with a sample of 15,575 women 15 to 49 years of age. Among these women, 7,717 were diagnosed with a chronic disease that required acquiring medicine and were considered eligible for the study. The dependent variable was defined as the diagnosis of a chronic disease and the need to obtain medication for treatment. Multivariate analysis used Poisson regression. Higher access was associated with residence in a rural area, having one or two chronic diseases, and higher socioeconomic status. Prevalence of access to medication was high, but the analysis revealed socioeconomic inequality in access to medicines in favor of the wealthy, while identifying the most vulnerable groups as the poorest and those with more chronic diseases.

Pharmaceitical Preparations;

Pharmacoepidemiology; Equity in Health; Cross-Sectional Studies
O objetivo deste trabalho foi analisar a prevalência de acesso a medicamentos para tratamento de doenças crônicas e a existência de desigualdades socioeconômicas no acesso. Os dados são da Pesquisa Nacional de Demografia e Saúde e da Mulher e da Criança de 2006, com uma amostra de 15.575 mulheres (15 a 49 anos). Dessas, 7.717 tiveram diagnóstico de doença crônica com necessidade de obtenção de medicamento e foram consideradas elegíveis para o estudo. $O$ desfecho foi construído com base no diagnóstico de doença crônica e na necessidade de obtenção de medicamento para o tratamento. A análise ajustada foi conduzida usando-se a regressão de Poisson. Os grupos que apresentaram maior prevalência de acesso foram os domiciliados na zona rural, com uma ou duas doenças crônicas e com nível socioeconômico mais elevado. A prevalência de acesso encontrada foi alta, no entanto, as análises demonstram que existe desigualdade socioeconômica no acesso a medicamentos a favor dos mais ricos, identificando como grupo mais vulnerável aquele dos indivíduos mais pobres e com maior número de doenças crônicas.

Preparações Farmacêuticas; Farmacoepidemiologia; Equidade em Saúde; Estudos Transversais 


\section{Introdução}

O acesso a medicamentos é um fator relevante no estudo da saúde pública, tendo em vista que é utilizado na análise do cumprimento do direito fundamental à saúde, sendo considerado um indicador de qualidade dos sistemas de saúde 1,2 . Algumas doenças crônico-degenerativas - como hipertensão arterial sistêmica, diabetes mellitus e problemas mentais - têm apresentado prevalência crescente no país, podendo este fato ser explicado principalmente pelo envelhecimento populacional 3,4 . Uma parte considerável dessas doenças tem como principal tratamento a administração de medicamentos de uso contínuo. A falta de acesso a esses medicamentos por parte da população é considerada como fator preponderante para determinar o aumento do número de retornos desses pacientes aos serviços de saúde 5 .

Segundo Arrais et al. 6, o acesso insuficiente aos medicamentos pode causar abandono do tratamento, com piora no estado de saúde e aumento do número de retornos aos serviços de saúde, ou um maior comprometimento da renda familiar com gastos com saúde. No entanto, considerando os sistemas de saúde públicos e privados, somente dois terços da população mundial têm acesso a tratamentos completos e eficazes 2 .

A Constituição Federal de 1988 define que a saúde é direito de todos e dever do Estado, devendo ser garantido o acesso universal e igualitário às ações e serviços de saúde. No Brasil, diversos trabalhos identificaram a existência de desigualdade socioeconômica em saúde, em que os grupos de renda mais elevada aparecem como aqueles que possuem maior acesso aos serviços de saúde $7,8,9$.

Desde a década de 1990, o Brasil tem buscado implementar políticas públicas visando a melhorar a disponibilização de medicamentos em quantidades adequadas às necessidades da população, sendo um dos poucos países onde o acesso gratuito a medicamentos essenciais é direito de todos os cidadãos. Entretanto, mesmo com a existência de um Sistema Único de Saúde (SUS) que assegura a assistência terapêutica integral incluindo a assistência farmacêutica, e no qual não há pagamento direto por parte do usuário, o gasto privado com medicamentos no país ainda é considerado expressivo 10 .

O objetivo central deste estudo foi analisar o acesso da população aos medicamentos, partindo da necessidade de uso dos mesmos e a existência de desigualdades socioeconômicas no acesso. Para isso, avaliou-se o acesso a medicamentos de uso contínuo para tratar hipertensão e doenças do coração; diabetes; bronquite e asma; problemas de saúde mental como depressão, ansiedade e insônia; anemia; artrite e reumatismo em uma amostra de mulheres.

\section{Métodos}

Para a realização deste trabalho foram utilizados os dados da Pesquisa Nacional de Demografia e Saúde da Mulher e da Criança (PNDS) de 2006 11, que está inserida na 5a fase do Projeto MEASURE DHS (Demographic and Health Survey). A DHS é um programa de escala global que tem como objetivo a análise e provimento de dados de países em desenvolvimento, focando na saúde e nutrição de mulheres e crianças. Em 2006, a estrutura tradicional dos questionários foi mantida, permitindo a comparabilidade nacional e internacional, mas incluiu temas que não haviam sido abrangidos anteriormente, como o acesso a medicamentos. Nessa última edição foram entrevistadas 15.575 mulheres com idades entre 15-49 anos (consideradas em idade fértil). A amostra é representativa das cinco macrorregiões do Brasil e de domicílios urbanos e rurais. $\mathrm{O}$ acesso a medicamentos foi definido com base no questionamento da existência de diagnóstico médico para alguma das doenças em estudo (hipertensão ou doenças do coração; diabetes; bronquite ou asma; problemas de saúde mental como depressão, ansiedade e insônia; anemia; artrite ou reumatismo). Em caso afirmativo, a entrevistada era questionada se o médico havia receitado medicamentos para tratar essas doenças alguma vez e se nos últimos trinta dias foi preciso obtê-los. A análise do acesso a medicamentos foi feita de forma dicotômica, considerando apenas aquelas que necessitaram obter o medicamento e classificando como acesso total as que obtiveram todos eles, e não acesso aquelas que obtiveram pelo menos um ou nenhum.

Como variáveis independentes foram analisadas: macrorregião administrativa de residência (Norte, Nordeste, Centro-oeste, Sudeste e Sul); área geográfica do domicílio (urbana e rural); faixa etária (15-19 anos, 20-39 e 40-49 anos); estado conjugal (solteira, casada e separada/viúva); cor da pele (branca ou não branca); anos de estudos (menos de 1 ano, 1-4, 5-8, 9-11 e 12 ou mais); nível socioeconômico (DHS Wealth Index, dividido em quintis); existência de cobertura por plano de saúde (sim ou não); e número de doenças crônicas diagnosticadas (1-2 e 3 ou mais). O DHS Wealth Index é um índice de riqueza construído para as pesquisas do projeto DHS que foi reproduzido para este estudo usando-se as questões relacionadas com bens e serviços já presentes no questionário domiciliar para outros fins. 
Adicionalmente, investigou-se como se deu a aquisição do medicamento (por intermédio do SUS, Farmácia Popular, farmácia comercial ou outras fontes) e, nos casos em que a entrevistada não conseguiu obter o medicamento, os motivos para não consegui-los (não estava disponível no SUS, não tinha na Farmácia Popular, não teve dinheiro para comprar, não teve tempo ou não sabia onde procurar, muito distante/não tem como ir).

As análises estatísticas foram realizadas no programa Stata/SE 12.0 (Stata Corp., College Station, Estados Unidos), considerando-se o efeito de delineamento amostral e os pesos individuais, por meio da utilização do conjunto de comandos svy, específico para a análise de inquéritos baseados em amostras complexas.

Dividiu-se a análise em três etapas. Num primeiro momento, apresenta-se a estatística descritiva do acesso a medicamentos em relação às variáveis independentes. Foi avaliada a prevalência de acesso total de acordo com as variáveis geográficas (macrorregião e área geográfica), demográficas (faixa etária, estado conjugal e cor da pele), socioeconômicas (anos de estudos e grupos de nível socioeconômico), organizacionais (cobertura de plano de saúde) e de saúde (número de doenças crônicas). Além disso, analisouse o acesso a medicamentos individualmente para cada doença crônica abordada no estudo, buscando avaliar a existência de diferenças entre os fatores associados ao acesso em cada uma das doenças. Sendo o foco do estudo verificar a existência de desigualdade socioeconômica, optou-se também por apresentar a prevalência de acesso a medicamentos, de acordo com as variáveis independentes, estratificada por nível socioeconômico. Foram incluídas razões de taxas de acesso entre as mais pobres (quintil inferior do índice de riqueza) em relação às mais ricas (quintil superior do índice de riqueza) para todas as categorias analisadas, como forma de observar a diferença relativa de acesso entre os grupos extremos de nível socioeconômico.

A segunda etapa corresponde à estimação do modelo usando-se regressão de Poisson para identificar aqueles fatores associados ao acesso a medicamentos. Os resultados são apresentados em razões de prevalências brutas e ajustadas para todas as variáveis incluídas no modelo e intervalos de 95\% de confiança (IC95\%). Na análise ajustada, as variáveis independentes incluídas no modelo foram macrorregião, área geográfica de residência, nível socioeconômico, faixa etária, estado conjugal, cor, anos de estudos, convênio de saúde e número de doenças. Entretanto, apenas aquelas com um nível de significância inferior a 0,05 após ajuste de todas as variáveis entre si foram mantidas no modelo final, isto é, área geográfica, nível socioeconômico e número de doenças.

Na terceira etapa foram conduzidas as análises secundárias, buscando descrever a forma de obtenção, quando houve acesso ao medicamento, e o motivo da não obtenção, quando não houve acesso. Além de incluir a porcentagem total para cada uma das opções, também é apresentada a análise estratificada por nível socioeconômico, de tal forma que seja possível identificar o perfil socioeconômico de cada fonte de obtenção de medicamentos. Foi considerada a possibilidade de mais de uma forma de obtenção, uma vez que alguns tratamentos exigem a utilização de mais de um medicamento, e a possibilidade de mais de um motivo para a falta de acesso. Os testes apresentados referem-se ao teste de Wald, para heterogeneidade, e de tendência linear para as variáveis ordinais, ambos considerando o ponto de corte de significância estatística de $5 \%$.

O projeto foi aprovado pelo Comitê de Ética em Pesquisa da Faculdade de Medicina da Universidade Federal de Pelotas, por meio do parecer no 535.270 .

\section{Resultados}

Dentre a amostra de 15.575 mulheres, 7.717 tiveram diagnóstico de doença crônica com necessidade de obtenção de medicamento e foram consideradas elegíveis para o estudo. A Tabela 1 descreve a composição da amostra e a prevalência de acesso aos medicamentos em relação às variáveis geográficas, demográficas, socioeconômicas, organizacionais e de saúde. A amostra foi dividida proporcionalmente entre as cinco macrorregiões do país, com $71 \%$ das pesquisadas domiciliadas na área urbana. A maioria tinha idades entre $20-39$ anos $(60,1 \%)$, era casada $(68,7 \%)$ e, em relação à cor da pele, $62,6 \%$ delas não se consideram brancas, tendo informado a cor da pele como preta, parda, amarela ou indígena. Um terço da amostra estudou de 9-11 anos (32,7\%). Em relação a convênios de saúde, 77,9\% das entrevistadas não possuíam plano de saúde, e dentre aquelas com diagnóstico de doença crônica, $87,6 \%$ relataram ter uma ou duas doenças.

A prevalência de acesso a medicamentos foi de 87,4\% (IC95\%: 85,2-89,5). Diferenças no acesso foram observadas em relação a estado conjugal, anos de estudos, nível socioeconômico, convênio de saúde e número de doenças. Identificou-se menor prevalência de acesso entre separadas/viúvas (80,3\%) quando comparadas às solteiras $(90,2 \%)$ e às casadas $(88 \%)$. As mulheres com escolaridade entre 5-8 anos tiveram menor 
Tabela 1

Descrição da amostra * e prevalência de acesso aos medicamentos de acordo com as variáveis geográficas, demográficas, socioeconômicas, organizacionais e de saúde. Pesquisa Nacional de Demografia e Saúde da Mulher e da Criança (PNDS), 2006, Brasil.

\begin{tabular}{|c|c|c|c|c|c|}
\hline \multirow[t]{2}{*}{ Variáveis } & \multicolumn{2}{|c|}{ Composição da amostra } & \multicolumn{3}{|c|}{ Acesso a medicamentos } \\
\hline & $\mathrm{n}$ & $\%$ & $\%$ & IC95\% & Valor de $p$ ** \\
\hline Total & 7.717 & 100,0 & 87,4 & $85,2-89,5$ & \\
\hline Macrorregião [ $n=7.717]$ & & & & & 0,4733 \\
\hline Norte & 1.497 & 19,4 & 84,5 & $79,6-89,4$ & \\
\hline Nordeste & 1.451 & 18,8 & 86,3 & $82,8-89,9$ & \\
\hline Sudeste & 1.628 & 21,1 & 87,3 & $83,3-91,2$ & \\
\hline Sul & 1.642 & 21,3 & 90,4 & $87,1-93,7$ & \\
\hline Centro-oeste & 1.499 & 19,4 & 88,2 & $84,7-91,6$ & \\
\hline Área geográfica [n=7.717] & & & & & 0,4723 \\
\hline Urbana & 5.482 & 71,0 & 87,1 & $84,7-89,5$ & \\
\hline Rural & 2.235 & 29,0 & 88,8 & $85,2-92,3$ & \\
\hline Faixa etária (anos) [n = 7.717] & & & & & 0,1583 \\
\hline $15-19$ & 872 & 11,3 & 80,8 & $70,9-90,8$ & \\
\hline $20-39$ & 4.637 & 60,1 & 87,7 & $84,9-90,4$ & \\
\hline $40-49$ & 2.208 & 28,6 & 88,5 & $85,7-91,4$ & \\
\hline Estado conjugal [n = 7.707] & & & & & 0,0194 \\
\hline Solteira & 1.396 & 18,1 & 90,2 & $85,4-94,9$ & \\
\hline Casada & 5.292 & 68,7 & 88,0 & $85,8-90,3$ & \\
\hline Separada/Viúva & 1.019 & 13,2 & 80,3 & $73,7-86,9$ & \\
\hline $\operatorname{Cor}[n=7.646]$ & & & & & 0,9499 \\
\hline Branca & 2.863 & 37,4 & 87,4 & $84,7-89,7$ & \\
\hline Não branca & 4.783 & 62,6 & 87,2 & $83,8-90,9$ & \\
\hline Anos de estudos [ $n=7.659]$ & & & & & 0,0365 \\
\hline Menos de 1 & 300 & 3,9 & 84,3 & $76,6-92,1$ & \\
\hline $1-4$ & 1.755 & 22,9 & 90,2 & $86,5-94,0$ & \\
\hline $5-8$ & 2.333 & 30,5 & 82,7 & $78,3-87,2$ & \\
\hline $9-11$ & 2.507 & 32,7 & 89,5 & $86,6-92,5$ & \\
\hline 12 ou mais & 764 & 10,0 & 89,2 & $82,6-95,9$ & \\
\hline Nível socioeconômico [n = 7.095] & & & & & 0,0005 \\
\hline 1ㅇ quintil (inferior) & 1.340 & 18,9 & 85,3 & $81,0-89,6$ & \\
\hline 2으 & 1.407 & 19,8 & 83,1 & $77,1-89,1$ & \\
\hline 3으 & 1.455 & 20,5 & 81,3 & $75,7-87,0$ & \\
\hline 4으 & 1.457 & 20,5 & 88,6 & $84,7-92,4$ & \\
\hline 5o quintil (superior) & 1.436 & 20,2 & 94,3 & $90,8-97,9$ & \\
\hline Convênio de saúde [n = 7.710] & & & & & 0,0331 \\
\hline Não & 6.005 & 77,9 & 85,8 & $83,3-88,3$ & \\
\hline Sim & 1.705 & 22,1 & 91,4 & $87,5-95,2$ & \\
\hline Número de doenças [n = 7.717] & & & & & 0,0054 \\
\hline 1 ou 2 & 6.763 & 87,6 & 88,6 & $86,2-91,0$ & \\
\hline 3 ou mais & 954 & 12,4 & 81,6 & $76,8-86,4$ & \\
\hline
\end{tabular}

IC95\%: intervalo de 95\% de confiança.

* Mulheres com diagnóstico de doença crônica (hipertensão/doenças do coração, diabetes, bronquite/asma, depressão/ ansiedade/insônia, anemia e artrite/reumatismo), indicação de uso e necessidade de obtenção de medicamentos nos últimos 30 dias;

** Teste qui-quadrado. 
acesso $(82,7 \%)$ em relação às outras categorias. Apresentaram maior acesso aquelas com maior nível socioeconômico (94,3\%), com posse de convênio de saúde $(91,4 \%)$ e uma ou duas doenças crônicas diagnosticadas $(88,6 \%)$ (Tabela 1$)$.

Ao analisar as prevalências de acesso a medicamentos para cada uma das doenças crônicas abordadas no estudo, observou-se que a prevalência foi maior para medicamentos usados no tratamento de hipertensão ou doenças do coração (93\%), quando comparada às outras doenças estudadas (prevalências de acesso variando de $85,5 \%$ a $90 \%$ ). Para hipertensão ou doenças do coração o acesso entre as mulheres com maior nível socioeconômico foi $13 \%$ mais alto em relação àquelas com menor nível socioeconômico, e $8 \%$ maior entre aquelas com uma ou duas doenças crônicas em relação às mulheres com maior número de doenças. Para o tratamento de bronquite e asma, as mais ricas indicaram acesso $15 \%$ superior em relação às mais pobres. O acesso a medicamento para depressão, ansiedade ou insônia diferiu de acordo com a macrorregião e nível socioeconômico, sendo que a Região Nordeste apresentou o menor acesso $(81,2 \%)$, e nas outras regiões variou de $88,1 \%$ a $92,9 \%$. O acesso para essas doenças foi $14 \%$ mais alto entre as mulheres com maior nível socioeconômico, em relação às mais pobres. No que se refere ao acesso a medicamentos para diabetes, anemia e para artrite e reumatismo, não foi encontrada diferença no acesso a medicamentos por macrorregião, área geográfica, nível socioeconômico e número de doenças (dados não apresentados em tabelas).

$\mathrm{O}$ acesso aos medicamentos aumenta com o incremento no nível socioeconômico. As mulheres incluídas no nível socioeconômico mais alto têm prevalência de acesso $11 \%$ maior do que aquelas do nível socioeconômico mais baixo. Entre as mulheres domiciliadas no Norte e Centrooeste, as prevalências de acesso foram $22 \%$ e $21 \%$ maiores entre as de nível socioeconômico mais alto, respectivamente. Em relação à área geográfica, entre as mulheres domiciliadas na zona urbana, o acesso foi $13 \%$ maior entre as mais ricas, quando comparadas às mais pobres. A maior diferença de acesso por nível socioeconômico foi observada entre as mulheres com maior número de doenças crônicas. Apresentar três ou mais doenças crônicas tendo menor nível socioeconômico é a situação de menor acesso dentre todas as categorias analisadas $(66,7 \%)$ e também é onde se observa a maior razão entre os grupos extremos de nível socioeconômico (Tabela 2).

Na Figura 1 é possível observar o impacto conjunto do nível socioeconômico e do número de doenças no acesso a medicamentos. Independentemente do número de doenças crônicas diagnosticadas, as mulheres com nível socioeconômico mais alto possuem maior acesso a medicamentos. No entanto, considerando apenas aquelas que apresentam uma ou duas doenças crônicas, a diferença de acesso entre as mais ricas e as mais pobres é de apenas $5,3 \%$, já entre as mulheres com três ou mais doenças crônicas observa-se que as mais ricas têm acesso 43,3\% maior quando comparadas às mais pobres.

Na Tabela 3, estão apresentados os resultados finais da análise ajustada, em que todas as variáveis em estudo fizeram parte do modelo, sendo que permaneceram associadas ao acesso a medicamentos no modelo final apenas as seguintes variáveis: área geográfica, nível socioeconômico e número de doenças. As mulheres domiciliadas na área rural apresentaram uma probabilidade $7 \%$ maior de acesso quando comparadas àquelas que residem na zona urbana. Mulheres pertencentes ao quintil superior de índice de bens apresentaram $13 \%$ mais probabilidade de acesso, em relação àquelas no quintil inferior. Ter um menor número de doenças indica uma probabilidade de acesso 7\% maior em relação a quem tem três ou mais doenças.

Considerando todas as entrevistadas que tiveram necessidade de obtenção de medicamentos, a maioria os obteve no SUS $(45,5 \%)$ ou em farmácias comerciais (50,8\%). Entre as mais pobres, a maior parcela obteve os medicamentos receitados no SUS $(66,3 \%)$, enquanto que $31,4 \%$ adquiriram o medicamento mediante pagamento em farmácias comerciais. Enquanto a obtenção na farmácia comercial aumenta com o incremento no nível socioeconômico, o acesso no SUS decresce. Entre as mais ricas, $76,7 \%$ obtiveram os medicamentos em farmácias comerciais, e apenas $17,2 \%$ recorreram ao SUS (Tabela 4 ).

Entre os motivos relatados para a falta de acesso, as respostas mais frequentes foram: "Não tinha no SUS" $(42,2 \%)$ e "Não tinha dinheiro" (30,5\%). Avaliando por nível socioeconômico, não ter o medicamento disponível no SUS foi o motivo relatado em maior número pelas mulheres incluídas no nível socioeconômico mais baixo (1o e 2 o quintis). O mesmo grupo também relatou mais vezes como motivo não ter dinheiro para comprar e ser muito longe/não tinha como ir buscar (dados não apresentados em tabelas).

\section{Discussão}

O presente estudo indicou uma alta prevalência de acesso a medicamentos e foram identificados como fatores associados ao acesso a área geográfica do domicílio, o nível socioeconômico e o número de doenças crônicas diagnosticadas. 
Tabela 2

Prevalência de acesso a medicamentos por variáveis geográficas, demográficas, socioeconômicas, organizacionais e de saúde, estratificada por nível socioeconômico. Pesquisa Nacional de Demografia e Saúde da Mulher e da Criança (PNDS) 2006, Brasil.

\begin{tabular}{|c|c|c|c|c|c|c|c|}
\hline \multirow[t]{3}{*}{ Variáveis } & \multicolumn{7}{|c|}{ Prevalência de acesso a medicamentos estratificada por nível socioeconômico } \\
\hline & 1o quintil & 2o quintil & 3o quintil & 4o quintil & 5o quintil & Valor de $p$ & Razão \\
\hline & & & & & & & Q5/Q1 \\
\hline Total & 85,3 & 83,1 & 81,3 & 88,6 & 94,3 & 0,0002 & 1,11 \\
\hline \multicolumn{8}{|l|}{ Macrorregião * } \\
\hline Norte & 77,9 & 88,5 & 78,3 & 93,8 & 94,7 & 0,0307 & 1,22 \\
\hline Nordeste & 86,3 & 83,8 & 90,8 & 84,9 & 90,5 & 0,5003 & 1,05 \\
\hline Sudeste & 90,6 & 77,4 & 69,0 & 88,4 & 94,5 & 0,0077 & 1,04 \\
\hline Sul & 85,0 & 86,0 & 90,6 & 91,3 & 93,6 & 0,1392 & 1,10 \\
\hline Centro-oeste & 81,5 & 88,6 & 83,9 & 88,0 & 98,5 & 0,0048 & 1,21 \\
\hline \multicolumn{8}{|l|}{ Área geográfica * } \\
\hline Rural & 89,3 & 91,3 & 87,4 & 87,5 & 96,9 & 0,9778 & 1,08 \\
\hline Urbana & 83,3 & 80,7 & 80,1 & 88,6 & 94,3 & 0,0001 & 1,13 \\
\hline \multicolumn{8}{|l|}{ Faixa etária (anos) ** } \\
\hline $15-19$ & 83,9 & 65,9 & 73,7 & 95,4 & 78,0 & 0,5659 & 0,93 \\
\hline $20-39$ & 86,2 & 86,8 & 81,3 & 86,6 & 94,7 & 0,0155 & 1,10 \\
\hline $40-49$ & 84,4 & 83,5 & 82,3 & 89,1 & 96,4 & 0,0001 & 1,14 \\
\hline \multicolumn{8}{|l|}{ Estado conjugal * } \\
\hline Solteira & 81,1 & 94,6 & 82,8 & 88,2 & 94,3 & 0,2023 & 1,16 \\
\hline Casada & 86,8 & 79,5 & 87,5 & 89,2 & 94,9 & 0,0002 & 1,09 \\
\hline Separada/Viúva & 79,5 & 90,5 & 56,5 & 85,7 & 91,2 & 0,2036 & 1,15 \\
\hline \multicolumn{8}{|l|}{ Cor * } \\
\hline Não branca & 85,2 & 78,6 & 86,5 & 89,1 & 95,4 & 0,0002 & 1,12 \\
\hline Branca & 85,5 & 92,2 & 73,0 & 87,7 & 93,3 & 0,0542 & 1,09 \\
\hline \multicolumn{8}{|l|}{ Anos de estudos ** } \\
\hline Menos de 1 & 80,2 & 78,7 & 97,6 & 88,3 & 100,0 & 0,1000 & 1,25 \\
\hline $1-4$ & 92,6 & 84,2 & 86,4 & 95,9 & 98,5 & 0,0864 & 1,06 \\
\hline $5-8$ & 76,4 & 79,5 & 77,6 & 86,1 & 95,2 & 0,0057 & 1,25 \\
\hline $9-11$ & 84,3 & 87,5 & 84,4 & 90,5 & 93,0 & 0,0403 & 1,10 \\
\hline 12 ou mais & 100,0 & 100,0 & 61,1 & 72,7 & 95,6 & 0,1443 & 0,96 \\
\hline \multicolumn{8}{|l|}{ Convênio de saúde * } \\
\hline Não & 84,5 & 81,2 & 81,8 & 90,2 & 92,5 & 0,0044 & 1,09 \\
\hline Sim & 98,9 & 99,1 & 79,0 & 84,4 & 95,4 & 0,2342 & 0,97 \\
\hline \multicolumn{8}{|l|}{ Número de doenças } \\
\hline \multicolumn{8}{|l|}{ crônicas ** } \\
\hline 1 ou 2 & 89,4 & 84,1 & 82,4 & 89,7 & 94,1 & 0,0132 & 1,05 \\
\hline 3 ou mais & 66,7 & 78,5 & 76,7 & 83,9 & 95,6 & 0,0006 & 1,43 \\
\hline
\end{tabular}

* Teste qui-quadrado;

** Teste de tendência.

Não se observou diferença no acesso segundo macrorregião, idade, estado conjugal, cor da pele, escolaridade e posse de convênio de saúde.

Utilizando-se a base de dados da PNDS 2006, pôde-se trabalhar com uma amostra com representatividade nacional, uma vez que refere-se a uma pesquisa domiciliar por amostragem probabilística complexa, que buscou avaliar mais de 15 mil mulheres e aproximadamente 5 mil crianças com idades até cinco anos, com representação nas cinco macrorregiões do país e incluindo os contextos urbano e rural.

A prevalência de acesso encontrada neste trabalho foi superior à encontrada por Paniz et al. 8 ao avaliar o acesso a medicamentos para a população do Sul e do Nordeste do país, que identifi- 
Figura 1

Prevalência de acesso a medicamentos entre grupos extremos de nível socioeconômico e número de doenças crônicas. Pesquisa Nacional de Demografia e Saúde da Mulher e da Criança (PNDS), 2006, Brasil.

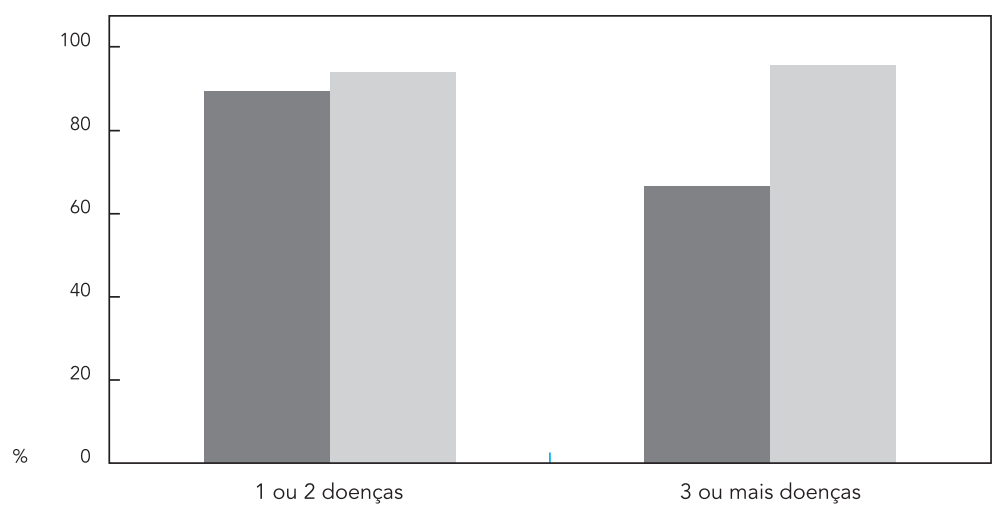

1ำ quintil de nível socioeconômico

$5^{\circ}$ quintil de nível socioeconômico

Tabela 3

Razões de prevalências (RP) brutas e ajustadas estimadas por meio de regressão de Poisson *. Pesquisa Nacional de Demografia e Saúde da Mulher e da Criança (PNDS), 2006, Brasil.

\begin{tabular}{|c|c|c|c|c|c|c|}
\hline \multirow[t]{2}{*}{ Variáveis } & \multicolumn{3}{|c|}{ Análise bruta } & \multicolumn{3}{|c|}{ Análise ajustada } \\
\hline & RP & IC95\% & Valor de $p$ & RP & IC95\% & Valor de $p$ \\
\hline Área geográfica ** & & & 0,4696 & & & 0,0210 \\
\hline Urbana & 1,00 & & & 1,00 & & \\
\hline Rural & 1,02 & $0,97-1,07$ & & 1,07 & $1,01-1,14$ & \\
\hline Nível socioeconômico *** & & & 0,0002 & & & 0,0000 \\
\hline 1ㅇ quintil (inferior) & 1,00 & & & 1,00 & & \\
\hline 2으 & 0,97 & $0,89-1,06$ & & 0,98 & $0,91-1,07$ & \\
\hline 3으 & 0,95 & $0,87-1,04$ & & 0,97 & $0,88-1,05$ & \\
\hline 4으 & 1,04 & $0,97-1,11$ & & 1,06 & $0,98-1,14$ & \\
\hline 5ㅇ quintil (superior) & 1,11 & $1,04-1,18$ & & 1,13 & $1,05-1,21$ & \\
\hline $\begin{array}{l}\text { Número de doenças } \\
\text { crônicas ** }\end{array}$ & & & 0,0131 & & & 0,0300 \\
\hline 1 ou 2 & 1,08 & $1,02-1,16$ & & 1,07 & $1,01-1,15$ & \\
\hline 3 ou mais & 1,00 & & & 1,00 & & \\
\hline
\end{tabular}

IC95\%: intervalo de 95\% de confiança.

* As variáveis incluídas no modelo foram ajustadas entre si, sendo apresentadas apenas aquelas que apresentaram valor de $p<0,05$. Não foram apresentados os resultados para as variáveis macrorregião, faixa etária, estado conjugal, cor, anos de estudo e convênio de saúde, por não apresentarem significância estatística na análise ajustada;

** Teste de Wald para heterogeneidade;

*** Teste de Wald para tendência linear.

cou entre adultos um acesso total de $81,2 \%$. No entanto, foi inferior ao resultado dos trabalhos de Aziz et al. 9, que descreveram a prevalência de acesso a medicamentos e os fatores associados para os idosos de Florianópolis, Santa Catarina, e de Bertoldi et al. 12, que descreveram o acesso e 
Tabela 4

Forma de obtenção dos medicamentos de acordo com o nível socioeconômico. Pesquisa Nacional de Demografia e Saúde da Mulher e da Criança (PNDS), 2006, Brasil.

\begin{tabular}{|c|c|c|c|c|c|c|}
\hline \multirow[t]{2}{*}{ Forma de obtenção (\%) } & \multicolumn{5}{|c|}{ Nível socioeconômico * } & \multirow[t]{2}{*}{ Todos } \\
\hline & 1o quintil & 2o quintil & 3의 quintil & 4o quintil & 5ㅇ quintil & \\
\hline SUS & 66,3 & 59,3 & 47,6 & 43,1 & 17,2 & 45,5 \\
\hline Outra grátis & 0,5 & 0,3 & 0,3 & 0,4 & 1,4 & 0,6 \\
\hline Farmácia comercial & 31,4 & 38,1 & 49,3 & 53,1 & 76,7 & 50,8 \\
\hline Farmácia Popular & 1,8 & 1,8 & 2,7 & 3,0 & 4,6 & 2,9 \\
\hline Outra paga & 0,0 & 0,5 & 0,1 & 0,4 & 0,1 & 0,2 \\
\hline Total & 100,0 & 100,0 & 100,0 & 100,0 & 100,0 & 100,0 \\
\hline
\end{tabular}

* Demographic and Health Survey (DHS) Wealth Index - índice de riqueza.

o uso de medicamentos, com base em dados de uma população coberta pelo Programa Saúde da Família (PSF) em Porto Alegre, Rio Grande do Sul, onde ambos identificaram prevalência de acesso de aproximadamente 95\%. Essa diferença pode ser consequência de características específicas das populações estudadas, possivelmente ligadas aos recortes de região, idade e cobertura pelo PSF, nas amostras utilizadas.

Considerando acesso e utilização de serviços de saúde, boa parte dos estudos identifica maiores prevalências entre os moradores da zona urbana 13,14 . No presente trabalho, o acesso a medicamentos para tratar doenças crônicas foi ligeiramente maior na zona rural quando comparada à zona urbana. Levando-se em conta o acesso a medicamentos por meio do SUS, Boing et al. 15 também identificaram, como resultado de análise bruta, uma pequena diferença de acesso em favor dos usuários residentes na zona rural. Esse resultado pode ser decorrente da diferença existente entre as prevalências das enfermidades estudadas. Avaliando a prevalência de doenças crônicas autorreferidas, Almeida et al. 16 identificaram que indivíduos que residem na zona rural têm uma prevalência de doenças crônicas $4 \%$ maior do que aqueles domiciliados na zona urbana.

A condição socioeconômica aparece como fator associado em diversos estudos que focaram o acesso e utilização de diferentes serviços de saúde, mostrando que uma maior renda ou um melhor nível socioeconômico está diretamente relacionado a uma maior probabilidade de utilização de serviços de saúde como consultas médicas e internação hospitalar 17, além de acesso e utilização de medicamentos 6,14,18. No presente trabalho, o nível socioeconômico também esteve associado ao acesso a medicamentos, uma vez que se observou que as mulheres mais ricas tiveram acesso $13 \%$ maior do que as mais pobres. Esse resultado confirma a existência de uma ligeira desigualdade socioeconômica no acesso a medicamentos, no entanto, considerando a disparidade na distribuição de renda existente no país ${ }^{19}$, a diferença de acesso a medicamentos entre ricos e pobres não é tão expressiva.

O número de doenças crônicas também foi identificado como fator associado ao acesso a medicamentos. Uma vez que o estudo avaliou mulheres que receberam diagnóstico de doença crônica, a análise foi feita em relação ao número de doenças diagnosticadas. O maior acesso foi observado entre as que tinham menor número de doenças crônicas. Apresentar menor número de doenças crônicas aumentou a probabilidade de ter acesso a todos os medicamentos necessários ao tratamento. Esse resultado pode estar relacionado ao fato de que, quanto maior o número de doenças maior a diversidade de medicamentos necessários para um tratamento completo. Dentre aqueles que pagam pelo medicamento, isso significa um maior comprometimento da renda e, dentre os que recebem o medicamento por meio do SUS, as possíveis ineficiências da assistência farmacêutica podem determinar que o indivíduo não consiga obter no sistema público todos os medicamentos de que necessita 20.

A associação desses dois fatores (socioeconômico e número de doenças crônicas) com o acesso a medicamentos indica uma realidade preocupante da assistência à saúde no Brasil. Além de boa parte da população depender do SUS para adquirir medicamentos, estudos apontam que algumas doenças crônicas apresentam maior prevalência entre os mais pobres 16,21. Levando-se 
em conta que a assistência farmacêutica ainda registra inúmeras falhas, não conseguindo atender integralmente a toda a população 20 , muitos desses pacientes podem ter dificuldades para manter um tratamento contínuo, sendo obrigados a comprometer uma parcela maior da renda familiar ou a abandonar o tratamento. Isso pode levar ao agravamento do estado de saúde, com consequente aumento de gastos com procedimentos mais caros como atendimento ambulatorial e internações hospitalares 6 .

De uma maneira geral, os gradientes de maior acesso foram no sentido dos grupos de maior nível socioeconômico, o que está de acordo com a literatura 12. A maior razão de taxas (5o quintil/1으 quintil) observadas foi entre as mulheres com três ou mais doenças crônicas diagnosticadas, indicando a situação de maior vulnerabilidade entre as que têm maior número de doenças e são mais pobres. Esse achado vai ao encontro da "Lei dos Cuidados Inversos" em saúde, que sugere que a disponibilidade de um bom tratamento médico tende a variar inversamente com a necessidade deste na população atendida 22 . Dessa forma, o resultado ressalta a necessidade de orientar as políticas públicas e a tomada de decisões à promoção da equidade no acesso aos medicamentos.

Utilizando o mesmo banco de dados, Perini 23 avaliou o acesso aos medicamentos estratificado por doença, considerando como acesso os casos em que o indivíduo obteve todos (acesso total) ou pelo menos um (acesso parcial) dos medicamentos receitados, o que justifica as diferenças encontradas em alguns resultados. $\mathrm{O}$ acesso a medicamentos para tratamento de diabetes, depressão, ansiedade e insônia, e anemia foi semelhante, já para hipertensão, bronquite e asma, e para artrite e reumatismo os fatores associados divergiram entre os estudos.

É possível verificar que há diferenças nos fatores associados ao acesso quando se analisa cada uma das doenças crônicas separadamente, provavelmente em função dos diferentes medicamentos necessários a cada tratamento. Esse fato demonstra a importância de políticas públicas que identifiquem as individualidades de cada demanda e executem ações que levem à diminuição das desigualdades.

Avaliando os aspectos metodológicos, devese considerar a existência de algumas limitações dos resultados deste trabalho. Há um potencial viés de memória que pode influenciar a informação a respeito de diagnósticos de doenças e prescrição de medicamento em algum momento, além da necessidade de obtenção nos trinta dias anteriores à entrevista. Além do problema de memória, é possível que a prevalência de doenças crônicas esteja subestimada devido à ausência de diagnóstico entre algumas mulheres da amostra.

Outro fator limitador diz respeito à amostra estudada. Uma vez que a PNDS inclui em sua amostra apenas mulheres em idade fértil (15-49 anos), não permite avaliar o acesso a medicamentos entre idosos, faixa etária mais acometida por doenças crônicas. Estudos identificaram uma grande prevalência de óbitos, entre pessoas com mais de 60 anos, relacionados a doenças crônico-degenerativas 24,25,26, mostrando a grande importância do acesso a medicamentos nessa faixa etária.

Apesar de a PNDS não incluir homens, a utilização de uma amostra exclusivamente feminina tem relevância para o trabalho sobre acesso a medicamentos, uma vez que historicamente as mulheres são consideradas responsáveis pela saúde da família e costumam cuidar mais de sua saúde quando comparadas aos homens ${ }^{27}$. As mulheres costumam buscar mais os serviços de saúde do que os homens 28,29, e estudos desenvolvidos no Brasil e no exterior demonstram que há uma maior utilização de medicamentos entre elas, independentemente da idade 6,18,30,31,32,33. Esses fatores demonstram a importância de elaborar estudos referentes ao acesso a medicamentos focando nessa parcela da população.

Conclui-se que pessoas mais pobres e com piores condições de saúde têm mais dificuldade de ter acesso a um tratamento completo e eficaz. Tratando-se de doenças crônicas degenerativas, a não utilização ou subutilização dos medicamentos necessários impossibilita um tratamento adequado, podendo levar ao agravamento das enfermidades ou complicações de saúde, aumentando a busca por outros serviços de saúde e os gastos com procedimentos mais dispendiosos. Isso mostra a necessidade de políticas públicas direcionadas à promoção da equidade, buscando levar a assistência farmacêutica àqueles com maiores dificuldades de acesso. 


\section{Resumen}

El objetivo de este estudio fue analizar la prevalencia del acceso a los medicamentos para enfermedades crónicas y la existencia de desigualdades socioeconómicas en su acceso a los mismos. Los datos son de la Encuesta Nacional de Demografía y Salud de la Mujer y la Infancia de 2006, con una muestra de 15.575 mujeres (15-49 años). Entre éstas, 7.717 fueron diagnosticadas de enfermedad crónica con necesidad de obtener medicamentos, y fueron elegibles para este estudio. Se construyó la variable dependiente, a partir del diagnóstico de la enfermedad crónica, y de la necesidad de obtener la medicación para el tratamiento. El análisis multivariado fue realizado mediante regresión de Poisson. Los grupos que tenían mayor prevalencia de acceso estaban domiciliados en una zona rural, con una o dos enfermedades crónicas y una mejor posición socioeconómica. La prevalencia encontrada de acceso fue elevada, sin embargo, el análisis muestra que existe una desigualdad socioeconómica en el acceso a los medicamentos, en favor del grupo más rico, se identificó el grupo más pobre como más vulnerable y con mayor número de enfermedades crónicas.

Preparaciones Farmacéuticas; Farmacoepidemiología; Equidad en Salud; Estudios Transversales

\section{Colaboradores}

F. Katrein participou da concepção do projeto, análise e interpretação dos dados, redação do artigo, revisão crítica relevante do conteúdo intelectual, aprovação da versão a ser publicada; é responsável por todos os aspectos do trabalho na garantia da exatidão e integridade de qualquer parte da obra. C. A. O. Tejada e A. D. Bertoldi colaboraram na concepção do projeto, interpretação dos dados, revisão crítica relevante do conteúdo intelectual, aprovação da versão a ser publicada; são responsáveis por todos os aspectos do trabalho na garantia da exatidão e integridade de qualquer parte da obra. M. C. Restrepo-Méndez contribuiu na análise e interpretação dos dados, revisão crítica relevante do conteúdo intelectual, aprovação da versão a ser publicada; é responsável por todos os aspectos do trabalho na garantia da exatidão e integridade de qualquer parte da obra.

\section{Referências}

1. Ferreira R. Consumo crônico de medicamentos na população de um Centro de Saúde. Rev Port Med Geral Fam 2007; 23:125-32.

2. Organización Mundial de la Salud. Acceso equitativo a los medicamentos esenciales: un marco para la acción colectiva. Genebra: Organización Mundial de la Salud; 2004.

3. Paniz VM, Fassa AG, Maia MF, Domingues MR, Bertoldi AD. Measuring access to medicines: a review of quantitative methods used in household surveys. BMC Health Serv Res 2010; 10:146.
4. Tasca RS, Soares DA, Cuman RKN. Acesso a medicamentos anti-hipertensivos em unidade básica de saúde em Maringá-Paraná. Arq Ciências Saúde UNIPAR 1999; 3:117-24.

5. Secretaria de Políticas de Saúde. Política nacional de medicamentos. Rev Saúde Pública 2000; 34:206-9.

6. Arrais PSD, Brito LL, Barreto ML, Coelho HLL. Prevalência e fatores determinantes do consumo de medicamentos no Município de Fortaleza, Ceará, Brasil. Cad Saúde Pública 2005; 21:1737-46. 
7. Fernandes LCL, Bertoldi AD, Barros AJD. Health service use in a population covered by the Estratégia de Saúde da Família (Family Health Strategy). Rev Saúde Pública 2009; 43:595-603.

8. Paniz VMV, Fassa AG, Facchini LA, Bertoldi AD, Piccini RX, Tomasi E, et al. Acesso a medicamentos de uso contínuo em adultos e idosos nas regiões Sul e Nordeste do Brasil. Cad Saúde Pública 2008; 24:267-80

9. Aziz MM, Calvo MC, Schneider IJC, Xavier AJ, d'Orsi E. Prevalência e fatores associados ao acesso a medicamentos pela população idosa em uma capital do sul do Brasil: um estudo de base populacional. Cad Saúde Pública 2011; 27:1939-50.

10. Bertoldi AD, Barros AJD, Camargo AL, Hallal PC, Vandoros S, Wagner A, et al. Household expenditures for medicines and the role of free medicines in the Brazilian public health system. Am J Public Health 2001; 101:916-21.

11. Berquó E, Garcia S, Lago TDG, organizadoras. Pesquisa Nacional de Demografia e Saúde da Criança e da Mulher - PNDS 2006: dimensões do processo reprodutivo e da saúde da criança. Brasília: Centro Brasileiro de Análise e Planejamento, Ministério da Saúde; 2009. (Série G. Estatística e Informação em Saúde).

12. Bertoldi AD, Barros AJD, Wagner A, Ross-Degnan D, Hallal PC. Medicine access and utilization in a population covered by primary health care in Brazil. Health Policy 2009; 89:295-302.

13. Viacava F, Travassos CM, Pinheiro RS, Brito A. Projeto: gênero e utilização de serviços de saúde no Brasil, financiado pela OPS. Relatório final de pesquisa. Rio de Janeiro: Departamento de Informações em Saúde, Centro de Informação Científica e Tecnológica, Fundação Oswaldo Cruz; 2001.

14. Travassos C. Eqüidade e o Sistema Único de Saúde: uma contribuição para debate. Cad Saúde Pública 1997; 13:325-30.

15. Boing AC, Bertoldi AD, Boing AF, Bastos JL, Peres KG. Acesso a medicamentos no setor público: análise de usuários do Sistema Único de Saúde no Brasil. Cad Saúde Pública 2013; 29:691-701.

16. Almeida MF, Barata RB, Montero CV, Silva ZP. Prevalência de doenças crônicas auto-referidas e utilização de serviços de saúde, PNAD/1998, Brasil. Ciênc Saúde Coletiva 2002; 7:743-56.

17. Andrade MV, Noronha K, Menezes RM, Souza MN, Reis CDB, Martins DR, et al. Desigualdade socioeconômica no acesso aos serviços de saúde no Brasil: um estudo comparativo entre as regiões brasileiras em 1998 e 2008. Economia Aplicada 2013; 17:623-45.

18. Bertoldi AD, Barros AJD, Hallal PC, Lima RC. Utilização de medicamentos em adultos: prevalência e determinantes individuais. Rev Saúde Pública 2004; 38:228-38.

19. Barros RP, Henriques R, Mendonça R. A estabilidade inaceitável: desigualdade e pobreza no Brasil. Rio de Janeiro: Instituto de Pesquisa Econômica Aplicada; 2001. (Texto para Discussão, 800).
20. Vieira FS. Assistência farmacêutica no sistema público de saúde no Brasil. Rev Panam Salud Pública 2010; 27:149-56.

21. Lessa I, Mendonça GA, Teixeira MT. Non-communicable chronic diseases in Brazil: from risk factors to social impact. Bol Oficina Sanit Panam 1996; 120:389-413.

22. Hart JT. The inverse care law. Lancet 1971; 297: 405-12.

23. Perini E. Acesso a medicamentos. In: Berquó E, Garcia S, Lago TDG, organizadoras. Pesquisa Nacional de Demografia e Saúde da Criança e da Mulher - PNDS 2006: dimensões do processo reprodutivo e da saúde da criança. Brasília: Centro Brasileiro de Análise e Planejamento, Ministério da Saúde; 2009. p. 279-95. (Série G. Estatística e Informação em Saúde).

24. Chaimowicz F. A saúde dos idosos brasileiros às vésperas do Século XXI: problemas, projeções e alternativas. Rev Saúde Pública 1997; 31:184-200.

25. Cabrera MAS, Andrade SM, Wajngarten M. Causas de mortalidade em idosos: estudo de seguimento de nove anos. Revista Geriatria \& Gerontologia 2007; 1:14-20.

26. Lima-Costa MF, Peixoto SV, Giatti L. Tendências da mortalidade entre idosos brasileiros (1980-2000). Epidemiol Serv Saúde 2004; 13:217-28.

27. Tezoquipa IH, Monreal MLA, Santiago RV. El cuidado a la salud en el ámbito doméstico: interacción social y vida cotidiana. Rev Saúde Pública 2001; 35:443-50.

28. Loyola Filho AI, Uchôa E, Guerra HL, Firmo JO, Lima-Costa MF. Prevalência e fatores associados à automedicação: resultados do projeto Bambuí. Rev Saúde Pública 2002; 36:55-62.

29. Mestanza F, Pamo O. Estudio muestral del consumo de medicamentos y automedicación en Lima metrópole. Rev Méd Hered 1992; 3:101-8.

30. Neutel CI, Walop W. Drug utilization by men and women: why the differences? Drug Inf J 2005; 39:299-310.

31. Ribeiro AQ, Rozenfeld S, Klein CH, César CC, Acurcio FA. Inquérito sobre o uso de medicamentos por idosos aposentados, Belo Horizonte, MG. Rev Saúde Pública 2008; 42:724-32.

32. Carvalho MF, Pascom ARP, Souza-Júnior PRB, Damacena GN, Szwarcwald CL. Utilization of medicines by the Brazilian population, 2003. Cad Saúde Pública 2005; 21 Suppl:S100-8.

33. Cabrita J, Ferreira HS, Iglesias P, Baptista TM, Rocha E, Silva LA, et al. Study of drug utilization among students at Lisbon University in Portugal. Pharmacoepidemiol Drug Saf 2002; 11:333-4.

Recebido em 28/Mai/2014

Versão final apresentada em 09/Dez/2014 Aprovado em 05/Fev/2015 